\title{
Measurement of Anticoagulation in Patients on Dabigatran, Rivaroxaban, and Apixaban Therapy by Novel Automated Thrombelastography
}

\author{
Ramin Artang ${ }^{1,20}$ Joao D. Dias ${ }^{3}$ Mark Walsh ${ }^{4}$ Kevin Bliden ${ }^{5}$ Jorn D. Nielsen ${ }^{2}$ Maren Anderson ${ }^{6}$ \\ Brian C. Thurston ${ }^{7}$ Udaya S. Tantry ${ }^{5}$ Jan Hartmann ${ }^{3}$ Paul A. Gurbel ${ }^{5}$ (1)
}

${ }^{1}$ Essentia Health St. Mary's Heart and Vascular Center, Duluth, Minnesota, United States

2 Bispebjerg University of Copenhagen Hospital, Department of Cardiology, Copenhagen, Denmark

3 Haemonetics Corp., Braintree, Massachusetts, United States

${ }^{4}$ Memorial Hospital of South Bend, Department of Energy Medicine, Sound Bend, Indiana, United States

${ }^{5}$ Sinai Center for Thrombosis Research and Drug Development, Sinai

Hospital of Baltimore, Baltimore, Maryland, United States

6 University of Minnesota School of Medicine, Duluth, Minnesota, United States

${ }^{7}$ Spartanburg Regional Medical Center, Division of Surgery,

Spartanburg, South Carolina, United States
Address for correspondence Ramin Artang, MD, Essentia Health St. Mary's Heart and Vascular Center, 407 East 3rd Street, Duluth, 55805 MN, United States

(e-mail: ramin_artang@yahoo.com).

\section{Abstract \\ Keywords \\ - clinical trials: oral anticoagulants \\ - coagulation inhibitors \\ - diagnosis management \\ - apixaban \\ - rivaroxaban \\ - dabigatran}

Background Direct-acting oral anticoagulants (DOACs) do not require monitoring. Measurement of DOAC effect would be useful in the event of bleeding, trauma, and thromboembolism while on anticoagulation. We evaluated the effectiveness of the investigational DOAC assays on the TEC ${ }^{\circledR} 6 \mathrm{~s}$ Hemostasis Analyzer to assess the anticoagulant effect of DOACs in patients treated for atrial fibrillation or deep vein thrombosis (DVT). Methods Patients on treatment for a minimum of 7 days with standard doses of dabigatran, rivaroxaban, and apixaban were included. DOAC plasma concentrations and $T E G^{\circledR} 6$ s Reaction (R)-time were measured and correlated. The sensitivity, specificity, and negative predictive value (NPV) of R-time to detect DOAC concentrations of $\geq 30, \geq 50$, and $\geq 100 \mathrm{ng} / \mathrm{mL}$ were calculated.

Results A total of 189 patients were included, $(n=50)$ on apixaban, $(n=62)$ on rivaroxaban, $(n=53)$ on dabigatran, and $(n=24)$ on no DOAC were studied. Using the direct thrombin inhibitor (DTI) channel, R-time demonstrated strong linear correlation with dabigatran levels $(r=0.93, p<0.0001)$. Using the antifactor Xa (AFXa) channel, $R$ time demonstrated strong nonlinear correlation with rivaroxaban and apixaban levels ( $r_{\mathrm{s}}=0.92$ and 0.84 , respectively, $p<0.0001$ for both). R-time revealed strong sensitivity and NPV in detecting low DOAC levels for the predefined concentrations.

Conclusion R-time measured by TEG ${ }^{\circledR} 6$ s DOAC-specific cartridge has a strong correlation with concentrations of the most commonly used DOACs with high sensitivity and NPV for detecting lower drug levels that are considered clinically relevant for patients in need of antidote, or prior to urgent surgery. Further studies to determine the relation of R-time to clinical outcomes are warranted. received

March 25, 2021

accepted after revision

November 4, 2021
DOI https://doi.org/

10.1055/a-1692-1415.

ISSN 2512-9465.

\section{(c) 2021. The Author(s).}

This is an open access article published by Thieme under the terms of the Creative Commons Attribution License, permitting unrestricted use, distribution, and reproduction so long as the original work is properly cited. (https://creativecommons.org/licenses/by/4.0/)

Georg Thieme Verlag KG, Rüdigerstraße 14, 70469 Stuttgart, Germany 


\section{Introduction}

Laboratory assessment of the anticoagulant effect of the DOACs remains a challenge, a decade after this class of anticoagulants entered the market. While routine monitoring of these agents is not warranted, the ability to detect anticoagulation may be an important clinical tool in certain situations, such as overdose, bleeding, and urgent surgery. ${ }^{1}$

The Thrombelastograph $\left(\mathrm{TEG}^{\circledR} 6 \mathrm{~s}\right)$ analyzer is a novel siteof-care test for global evaluation of hemostasis. The TEG6s applies resonance-frequency viscoelasticity measurements and premixed disposable multichannel microfluidic cartridges. ${ }^{2}$ The DOAC-specific cartridge for the TEG ${ }^{\circledR} 6$ s system is an experimental prototype that was proven more sensitive and specific than the conventional Global Hemostasis cartridge in identifying the anticoagulant effect from DOAC therapy comparing DOAC treated patients to healthy volunteers without anticoagulation. ${ }^{3}$ A study on healthy volunteers revealed a significant correlation between the TEG Reaction time (R-time) and blood DOAC levels using this cartridge. ${ }^{4}$ In a recent study, the normal reference ranges for the DOAC-specific cartridge, and its effectiveness in detecting and classifying the DOAC treatment were established using blood samples from 160 healthy patients and 190 patients on treatment for atrial fibrillation and venous thromboembolism. ${ }^{5}$ The clinically relevant DOAC concentration cut-offs, based on current available literature, are $30 \mathrm{ng} / \mathrm{mL}$ for urgent invasive procedures with high bleeding risk, $50 \mathrm{ng} / \mathrm{mL}$ for antidote administration, ${ }^{6,7}$ and $100 \mathrm{ng} / \mathrm{mL}$ for thrombolysis in stroke. ${ }^{8}$ The purpose of this present study was to demonstrate the correlation between the R-time and the DOAC plasma concentrations, as well as to assess sensitivity, specificity, and negative predictive value (NPV) of the R-time for the aforementioned clinically useful DOAC concentrations in the same cohort of patients.

\section{Materials and Methods}

This study was conducted at the following five clinical sites in the United States from August 2016 until September 2017: Essentia Institute of Rural Health, Duluth, Minnesota, United States; Memorial Hospital of South Bend, South Bend, Indiana, United States; Spartanburg Regional Medical Center, Spartanburg, South Carolina, United States; Inova Heart and Vascular Institute, Falls Church, Virginia; and Inova Cardiology Ambulatory Research Center, Manassas, Virginia, United States. In accordance with the principles of Good Clinical Practice and the Declaration of Helsinki, Institutional Review Boards at each participating site conferred ethical approval. All participants were 18 years or older and gave informed consent prior to the enrollment.

For the on-DOAC group, patients were included if they were 18 years or older, and on a DOAC at doses recommended by the manufacturers for treatment of atrial fibrillation, venous thromboembolism, or thromboembolism prophylaxis for minimum of 7 days uninterrupted. The exclusion criteria were genetic bleeding disorders, known, or subsequently discovered inherited defects of coagulation (e.g., hemophilia or the von Willebrand disease), DOAC dosage outside of manufacturer's recommended range (e.g., study subject with renal impairment and supratherapeutic dose), heparin or low molecular weight heparin (LMWH) administered within 7 days prior to blood draw, on any other type on Food and Drug Administration (FDA) approved or experimental anticoagulant, bruising, wounds, or scarring in the area of venipuncture. Treatment with aspirin was not an exclusion criteria.

Blood samples were collected at a random time point at patient presentation to outpatient clinic or during inpatient presentation. A non-DOAC control group of patients was included in the analysis for comparison. Patients were included if they were 18 years or older. The exclusion criteria for the non-DOAC group were medical evidence of atrial fibrillation, deep vein thrombosis, or pulmonary embolism requiring anticoagulation, genetic bleeding disorders, known or subsequently discovered inherited defects of coagulation (e.g., hemophilia or the von Willebrand disease) on any medication containing heparin or LMWH within 7 days, a DOAC or other anticoagulant, any medications known to affect coagulation status, strict vegan diet, bruising, wounds, or scarring in the area of venipuncture.

\section{Blood Sampling and Analysis}

Up to approximately $20 \mathrm{~mL}$ of blood was drawn standard venipuncture from each patient. No restrictions were made on use of tourniquet. The first 3 to $6 \mathrm{~mL}$ nonadditive tube was discarded at each draw. The second tube of $4.5 \mathrm{~mL}$ was collected in a standard 3.2\% sodium citrate tube (BD Vacutainer, Franklin Lakes, New Jersey, United States), and was used for the TEG ${ }^{\circledR} 6 \mathrm{~s}$ analysis. The third tube of $4.5 \mathrm{~mL}$ was collected in a standard $3.2 \%$ sodium citrate tube and then centrifuged at $2,000 \mathrm{~g}$ for 10 minutes. The plasma samples were stored at $-80^{\circ} \mathrm{C}$ for the measurement of DOAC concentrations. DOAC concentrations were measured by High Performance Liquid Chromatography/Tandem Mass Spectrometry (LC-MS/MS) assay at Quest Diagnostics (Wood Dale, Illinois, United States). ${ }^{9}$

\section{Thrombelastography}

The Thrombelastograph ${ }^{\circledR} 6 s$ (TEG ${ }^{\circledR} 6 s$, Haemonetics Corp, Boston, Massachusetts, United States) technique has previously been described in detail. ${ }^{2,3}$ The TEG ${ }^{\circledR} 6$ s system measures the clot viscoelasticity using resonance-frequency. The blood sample suspended in a microring structure within the cartridge is exposed to a fixed vibration frequency. With LED illumination, a detector measures vertical motion of the blood film. The frequency leading to resonance is identified and then converted to the TEG ${ }^{\circledR}$ system readout. The DOACspecific cartridge contained kaolin in channel 1 (the citrated kaolin [CK] channel), ecarin in channel 2 (the direct thrombin inhibitor [DTI] channel), factor Xa (FXa) in channel 3 (the anti-FXa [AFXa] channel), and kaolin with abciximab in channel 4 (the functional fibrinogen channel). Based on unpublished pilot studies on patients treated with warfarin, the R-time was not altered beyond the established reference range for both DTI and AFXa channels with INRs ranging 
between 1.2 and 3.5. Heparin increased the R-time in the AFXa channel in a dose-dependent fashion but caused no change of R-time in the DTI channel. Experience with LMWH is pending and is considered as necessary part of the product development, but we anticipate similar response to the $\mathrm{R}$ time on the AFXa and DTI channels as heparin given its effect on FXa inhibition. The DOAC specific cartridge is an experimental prototype that is currently not commercially available and is under investigation for use in patients who are treated with DOACs. The current protocol for the TEG ${ }^{\circledR} 6 \mathrm{~s}$ system is to allow the samples to stabilize for 10 minutes prior to analysis, unless the analysis is needed urgently for medical emergency. In the present study, all the R-time analyses were performed between 10 minutes and 2 hours after the phlebotomy.

\section{Statistical Analysis}

The importance of measuring the R-time in patients treated with DOACs has been previously demonstrated. ${ }^{3,4}$ We determined the correlation between R and DOAC concentrations. Pearson's correlation coefficient $r$ was calculated for linear association between R-times and DOAC concentration. Spearman's rank correlation $r_{s}$ was calculated if the association of Rtimes and DOAC concentrations was nonlinear. Correlation coefficient values of $>0.8$ were considered strong. A $p$-value of $<0.05$ was considered statistically significant. If the association pattern did not fit a linear model, the best fit nonlinear model was selected. Goodness of fit expressed by $R^{2}$ was calculated for both linear and nonlinear regressions. Concentrations of rivaroxaban, apixaban, and dabigatran were classified into three categories as follows: (1) $<30$ versus $\geq 30$, (2) $<50$ versus $\geq 50$, and ( 3$)<100$ versus $\geq 100 \mathrm{ng} / \mathrm{mL}$. Logistic regression models compared R-times to the binary concentrations of ( $\geq 30, \geq 50$, and $\geq 100 \mathrm{ng} / \mathrm{mL}$ ) to identify initial cutoff points to initiate the detailed analysis of sensitivity, specificity, positive predictive value (PPV), negative predictive value (NPV), positive likelihood ( $\mathrm{LR}+$ ), and negative likelihood ratios (LR - ). Analysis of sensitivity, specificity, PPV, NPV, LR + , and LR- began at the R-time cut-off point identified by the logistic regression and continued for the next six R-times, consecutively. The best model fit was identified as the model with the highest LR+ and lowest LR- levels. ${ }^{10}$ The rational for the sample size was previously described. ${ }^{5}$ The correlations and graphs were generated using Prism software version 9 (GraphPad SoftwInc., La Jolla, California, United States). The sensitivity data were analyzed using IBM SPSS statistics (Version 23) predictive analytics software (IBM Corp. Armonk, New York, United States Released 2015).

\section{Results}

A total of 189 patients were included in the study. Of them, 165 were in the on DOAC group and 24 patients were included on the non-DOAC group. The clinical characteristics for each DOAC and non-DOAC groups are outlined in -Table 1. Among the patients on DOAC, 50 were on apixaban, 53 on rivaroxaban, and 62 on dabigatran. The mean age of DOAC cohort was $71 \pm 10.2$ years and $42 \%$ were female. Among patients not anticoagulated, the mean age was $48 \pm 11.6$ years and $67 \%$ were female. Using the DTI channel, R-time demonstrated a strong linear correlation with dabigatran levels $\left(r=0.93, p<0.0001\right.$, and $R^{2}=0.86$ for goodness of fit). Using the AFXa channel, R-time demonstrated strong nonlinear correlation with rivaroxaban and apixaban levels $\left(r_{s}=0.92\right.$ for rivaroxaban, $r_{s}=0.84$ for apixaban, $p<0.0001$, and $R^{2}=0.86$ for goodness of fit for both; - Fig. 1 ).

The DOAC levels among the non-DOAC group were 0 . Among the 50 patients on apixaban, one patient had a levels of $29 \mathrm{ng} / \mathrm{mL}$ or less, one patient between 30 and $49 \mathrm{ng} / \mathrm{mL}$, and six patients between 50 and $99 \mathrm{ng} / \mathrm{mL}$. Among the 53 patients on rivaroxaban, 4 had a level of $29 \mathrm{ng} / \mathrm{mL}$ or less, 7 had between 30 and $49 \mathrm{ng} / \mathrm{mL}$, and 17 had between 50 and $99 \mathrm{ng} / \mathrm{mL}$. Among the 62 patients on dabigatran, 2 had a level of $29 \mathrm{ng} / \mathrm{mL}$ or less, 9 had between 30 and $49 \mathrm{ng} / \mathrm{mL}$, and 17 had between 50 and $99 \mathrm{ng} / \mathrm{mL}$. The sensitivity, specificity, LR + , LR - , and NPVs for the R-time to detect DOAC concentrations of $\geq 30, \geq 50$, and $\geq 100 \mathrm{ng} / \mathrm{mL}$ for the entire cohort of on-DOAC and non-DOAC patients are presented in - Table 2 . R-time revealed strong sensitivity and NPV for the predefined DOAC concentrations. Due to only single-acquired concentration between 30 and $49 \mathrm{ng} / \mathrm{mL}$ in the apixaban group, no sensitivity and specificity calculations were performed in that range.

\section{Discussion}

To date, this is the largest study correlating the DOAC concentrations to TEG-R value using the DOAC-specific cartridge with the $\mathrm{TEG}^{\circledR} 6 \mathrm{~s}$ device. In this study, a strong correlation between R-time and DOAC concentration in 165 patients on DOAC was demonstrated. The R-time also revealed high sensitivity and NPV for the low doses of the three most commonly used DOAC agents.

This technology may be useful in clinical situations requiring rapid assessment of the coagulation status in patients who present with acute bleeding while on DOAC treatment. Other clinical situations where such test may prove to be useful are patients on DOAC needing urgent surgery, and prior to DCcardioversion, or radiofrequency ablation for patients with atrial fibrillation when there is uncertainty about the hemostatic function or adequacy of anticoagulation. The R-time assessment test duration was under 6 minutes.

The association observed between R-time, as well as the concentration of the FXa inhibitors rivaroxaban and apixaban, was best fitted in a nonlinear pattern, as increasing concentrations of the FXa inhibitor concentrations would not cause further increase of the R-time displaying a plateau in the curve (-Fig. 1B, C). We hypothesize that this observation may be related to the fixed concentration of FXa used in the FXa channel chamber. Alternative explanation may be the fixed amount of FXa in the whole blood sample that is present in the chamber, thus limited number of FXa binding sites for the FXa inhibitor molecules. It is therefore plausible that at higher concentrations of FXa inhibitor ( $>150-200 \mathrm{ng} / \mathrm{mL}$ ), a saturation point may be achieved where higher levels of the in vivo FXa inhibitor concentrations would no longer impact 
Table 1 Subjects clinical characteristics

\begin{tabular}{|c|c|c|c|c|}
\hline & Apixaban & Dabigatran & Rivaroxaban & Non-DOAC \\
\hline & $n=50$ & $n=62$ & $n=53$ & $n=24$ \\
\hline Mean age in years (SD) & $72(10.3)$ & $70.9(9.1)$ & $70.8(11.9)$ & $48(11.6)$ \\
\hline \multicolumn{5}{|l|}{$\begin{array}{l}\text { Gender } \\
n(\%)\end{array}$} \\
\hline Female & $25(50)$ & $24(39)$ & $25(47)$ & $16(67)$ \\
\hline Male & $25(50)$ & $38(61)$ & $28(53)$ & $8(33)$ \\
\hline \multicolumn{5}{|l|}{$\begin{array}{l}\text { Ethnicity } \\
n(\%)\end{array}$} \\
\hline Caucasian & $44(88)$ & $59(95)$ & $49(92)$ & $21(88)$ \\
\hline Other & $6(12)$ & $3(5)$ & $4(8)$ & $3(12)$ \\
\hline \multicolumn{5}{|l|}{$\begin{array}{l}\text { DOAC indication } \\
n(\%)\end{array}$} \\
\hline Atrial fibrillation or flutter & $43(86)$ & $62(100)$ & $45(85)$ & \\
\hline DVT/PE treatment & $4(8)$ & 0 & $6(11)$ & \\
\hline DVT prophylactic & $3(6)$ & 0 & $2(4)$ & \\
\hline \multicolumn{5}{|l|}{$\begin{array}{l}\text { Comorbidities } \\
n(\%)\end{array}$} \\
\hline Hyperlipidemia & $27(54)$ & $37(60)$ & $24(45)$ & $5(21)$ \\
\hline Hypertension & $38(76)$ & $43(69)$ & $33(62)$ & $10(42)$ \\
\hline Heart failure & $14(28)$ & $7(11)$ & $6(11)$ & \\
\hline Diabetes & $12(24)$ & $16(26)$ & $8(15)$ & \\
\hline Hypothyroidism & $4(8)$ & $8(13)$ & $6(11)$ & $1(4)$ \\
\hline Coronary artery disease & $3(6)$ & $6(10)$ & $10(19)$ & \\
\hline \multicolumn{5}{|l|}{$\begin{array}{l}\text { Antiplatelet therapy ConMed } \\
n(\%)\end{array}$} \\
\hline Aspirin & $9(18)$ & $17(27)$ & $10(19)$ & $2(8)$ \\
\hline Clopidogrel & $4(8)$ & $1(2)$ & $1(2)$ & \\
\hline Prasugrel & & & $1(2)$ & \\
\hline
\end{tabular}

Abbreviations: DOAC, direct-acting oral anticoagulants; DVT, deep vein thrombosis; PE, pulmonary embolism; SD, standard deviation.

the effect of FXa in converting prothrombin to thrombin and thereby fibrin generation. Since the R-time reflects the beginning of fibrin-platelet clot generation, the mechanism described above may explain the plateau in R-time with further increase of the FXa inhibitor concentration in the present study. This hypothesis requires further investigation with escalating FXa dose in in vitro samples analyzed. The correlation of dabigatran concentrations and R-time was linear in the concentrations observed in this study.

The routine monitoring of patients on chronic DOAC treatment, without thrombotic or bleeding events, is not recommended by the guidelines. ${ }^{11,12}$ The challenge for the clinicians is how to respond to a measured level that falls at the upper or lower end of a very wide range reported in patients on chronic DOAC treatment. ${ }^{13}$ Secondary analysis of the randomized DOAC trials has revealed association of higher drug concentration with bleeding and lower concentrations with thrombotic events. There is so far, no evidence that adjustment of the DOAC dose, beyond what was demonstrated in the original DOAC trials and stated in the prescription information, would improve the clinical outcome. Patients' propensity for thrombosis or bleeding may differ significantly based on patient characteristics including but not limited to age and renal function. It is therefore possible that the same concentration of DOAC in plasma can result in adequate protection in some patients but thrombosis or bleeding in others. ${ }^{14}$ The global coagulation assay parameters, such as the R-time in Thrombelastography and lag-time in thrombin generation assay, have a statistically significant correlation with DOAC concentration levels but do not offer an exact prediction of the DOAC concentration. ${ }^{4,15}$ The latter observation is further confirmed as seen by the scatter in -Fig. 1 in the present study. Our observation reveals that the strength of the TEG ${ }^{\circledR} 6$ s technique beyond its short turnaround time may be its high sensitivity and NPV for the predefined DOAC concentrations in a qualitative binary pattern ( $\geq 50$ or $100 \mathrm{ng} / \mathrm{mL}$ of drug present vs. no clinically significant amount present) rather than quantitative $(\mathrm{ng} / \mathrm{mL})$ assessment of DOAC concentrations. For concentrations of $30 \mathrm{ng} / \mathrm{mL}$ or lower and for potential clinical application of this technology in general, these observations will need to 


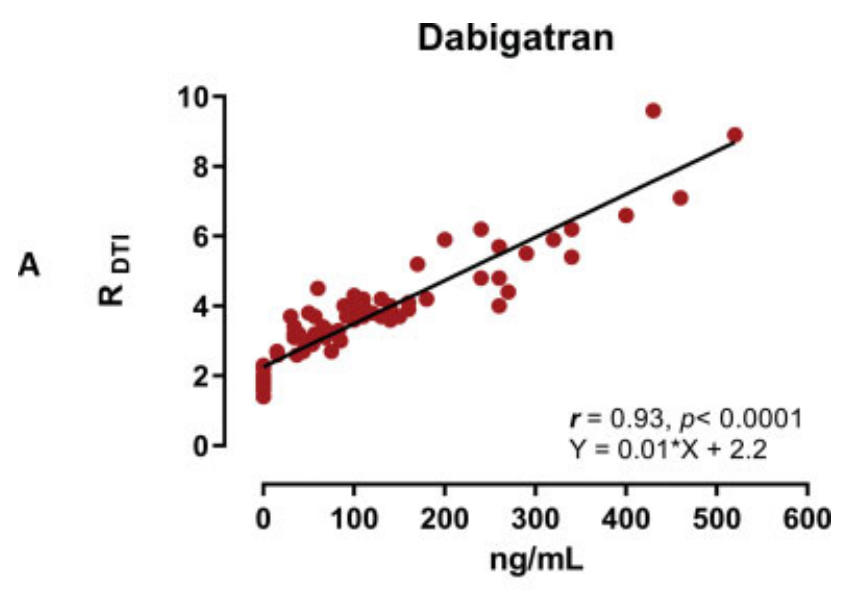

Rivaroxaban

B
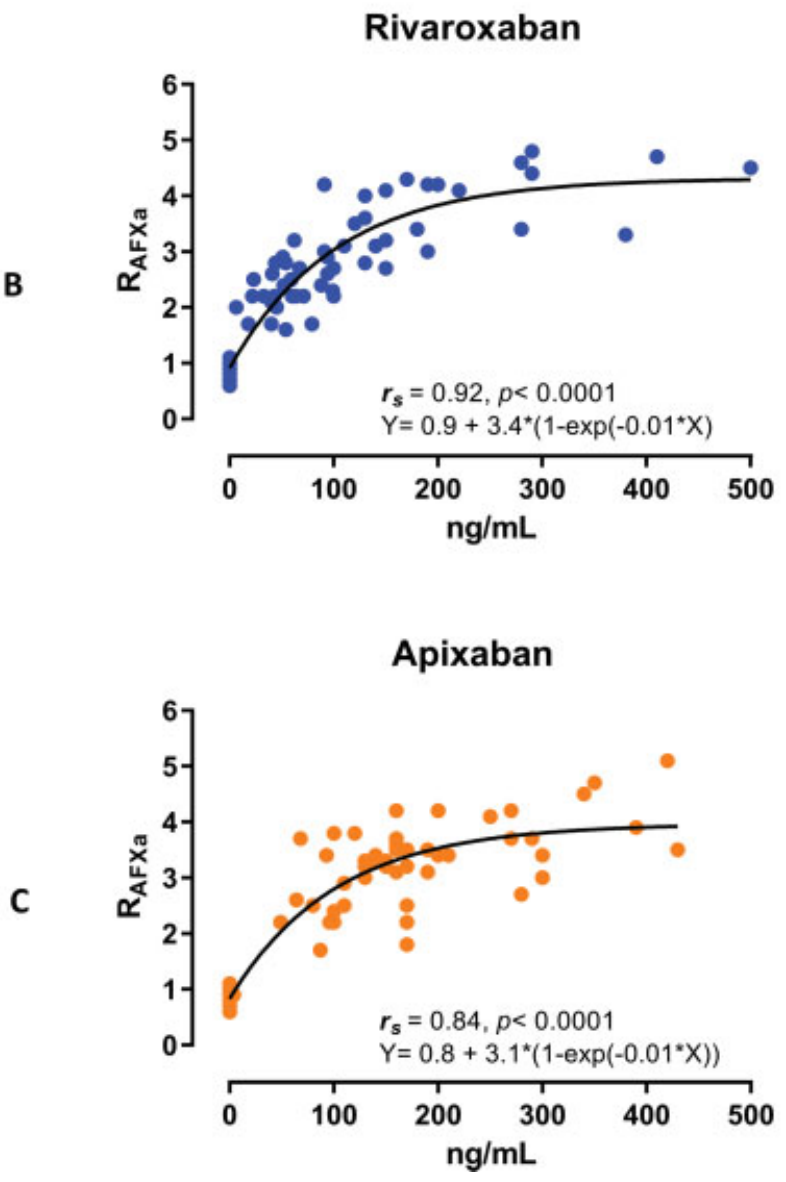

Fig. 1 (A-C) The scatter diagram of DOAC concentrations against Rtime. $R_{\mathrm{DTI}}$ indicates using the DTI channel; $R_{\mathrm{FXa}}$ indicates using the AFXa channel. AFXa, antifactor-Xa; DOAC, direct-acting oral anticoagulants; DTI, direct thrombin inhibitor; R, reaction time.

be verified with much larger cohorts and more robust and consistent high sensitivity and NPVs across different predefined low concentrations. The present study was not designed to answer the question of clinical outcomes correlation with Rtimes but may be considered as hypothesis generating research. Establishing such correlation is therefore warranted using the results from large clinical studies, associating DOAC concentrations and different coagulation parameters, includ- ing the global assays with the clinical outcomes or conducting new studies where the global assays are included.

\section{Limitations}

The limitation of the present study includes a lack of adequate data on edoxaban, the other currently FDA approved FXa inhibitor. Given only four patients included edoxaban group, no meaningful analysis could be performed on the data which were omitted from this manuscript. The thresholds achieved in the present study on rivaroxaban and apixaban can therefore not be used or deduced for edoxaban. The study design did not include hour-by-hour monitoring of the DOAC level but rather approaching the patients consecutively as they presented to the clinic or the hospital, regardless of the time of DOAC intake. The sensitivity and specificity calculations on apixaban for levels between 30 and $49 \mathrm{ng} / \mathrm{mL}$ were therefore less certain. A larger number of observations at DOAC levels less than $50 \mathrm{ng} / \mathrm{mL}$ would have further strengthen the sensitivity and specificity of this technique in lower concentrations where it considered clinically relevant. The study would furthermore have demonstrated much higher specificity, $\mathrm{NPV}$, and LR+ if the non-DOAC cohort was larger by design providing a larger true negative value.

\section{Conclusion}

In conclusion, R-time measured by TEG6s DOAC-specific cartridge has a strong correlation with concentrations of the most commonly used DOACs. This technique has high sensitivity and NPV for detecting lower drug levels that are considered clinically relevant for potential use in patients in need of antidote or prior to urgent surgery. Further larger studies with focus on lower concentrations and the relation of R-time to clinical outcomes are warranted. This novel assay has promise in the personalization of antithrombotic therapy in patients with cardiovascular and thrombotic diseases.

\section{What Is Known About This Topic?}

- Prior studies on the Thrombelastograph TEG ${ }^{\circledR} 6 s$ have shown favorable correlation between direct oral anticoagulants (DOACs) concentrations and TEG $^{\circledR}$ Reaction-time in small number of healthy volunteers.

\section{What Does This Paper Add?}

- We studied the correlation of $\mathrm{TEG}^{\circledR} 6 \mathrm{~s}$ Reaction (R)time to DOAC plasma concentrations in 165 patients on chronic DOAC treatment.

- $\mathrm{TEG}^{\circledR} 6 \mathrm{~s}$ R-time demonstrated highly significant correlation with dabigatran, rivaroxaban, and apixaban blood concentrations.

- The R-time demonstrated high sensitivity and negative predictive value to detect DOAC concentrations of $\geq 30$, $\geq 50$, and $\geq 100 \mathrm{ng} / \mathrm{mL}$. 
Table 2 Sensitivity, specificity and negative predictive value of R-time to detect DOAC concentrations above thresholds mentioned

\begin{tabular}{|l|l|l|l|l|l|l|l|}
\hline \multirow{2}{*}{ DOAC } & Threshold & R-time & Sensitivity & Specificity & LR+ & LR- & NPV \\
\cline { 2 - 8 } & $\geq \mathbf{n g} / \mathbf{m L})$ & $\geq(\mathbf{m i n})$ & $\%$ & $\%$ & & & $\%$ \\
\hline Dabigatran & & & & & & & \\
\hline & 30 & 2.6 & 100 & $92(82-100)$ & 13 & 0 & 100 \\
\hline & 50 & 3.1 & $94(88-100)$ & $83(70-95)$ & 5.5 & 0.07 & $91(81-100)$ \\
\hline & 100 & 3.4 & 100 & $82(72-93)$ & 5.6 & 0 & 100 \\
\hline Rivaroxaban & & & & & & & \\
\hline & 30 & 1.7 & $98(94-100)$ & $86(73-99)$ & 6.9 & 0.02 & $96(88-100)$ \\
\hline & 50 & 2.1 & $95(89-100)$ & $80(67-93)$ & 4.8 & 0.06 & $93(84-100)$ \\
\hline & 100 & 2.6 & $96(78.9-99.9)$ & $85(72.4-93.3)$ & 5.1 & 0.05 & $98(93-100)$ \\
\hline Apixaban & & & & & & & \\
\hline & 30 & $c$ & & & & & \\
\hline & 50 & 1.7 & 100 & $96(88-100)$ & 25 & 0 & 100 \\
\hline & 100 & 2.2 & $98(93-100)$ & $81(67-95)$ & 5 & 0.03 & $96(89-100)$ \\
\hline
\end{tabular}

Abbreviations: AFXa, antifactor-Xa; AUC, area under the curve; DOAC, direct oral anticoagulant; DTI, direct thrombin inhibitor; LR +, positive likelihood ratio; LR - , negative likelihood ratio; NPV, negative predictive value; R, reaction time.

Note: The reference range of R-time for the FXa channel is 0.6-1.5 minutes, and for DTI channel is 1.6-2.5 minutes.

andicates using the DTI channel.

bIndicates using the AFXa channel.

'Due to only single acquired concentration between 30 and $49 \mathrm{ng} / \mathrm{mL}$, no calculations were performed.

\section{Authors' Contributions}

R.A. and J.H. conceived and designed the study. M.W., K.B., B.C.T., M.A., J.D.N., and P.A.G. contributed to data collection and interpretation. All authors revised the article for important intellectual content and approved the final version for submission.

\section{Conflict of Interests}

J.D.D. and J.H. were employees of Haemonetics Corporation at the time of the study. This study was supported by Haemonetics Corporation (Boston, Massachusetts, United States). The other authors have no other relevant financial interest in the products or companies described in this article.

Funding

This study was funded by the Haemonetics Corporation.

\section{Acknowledgments}

We would like to thanks Rebecca Starr for her assistance with the language of the manuscript.

\section{References}

1 Douxfils J, Ageno W, Samama CM, et al. Laboratory testing in patients treated with direct oral anticoagulants: a practical guide for clinicians. J Thromb Haemost 2018;16(02):209-219

2 Gurbel PA, Bliden KP, Tantry US, et al. First report of the point-ofcare TEG: a technical validation study of the TEG-6S system. Platelets 2016;27(07):642-649

3 Bliden KP, Chaudhary R, Mohammed N, et al. Determination of non-vitamin $\mathrm{K}$ oral anticoagulant (NOAC) effects using a new- generation thrombelastography TEG 6s system. J Thromb Thrombolysis 2017;43(04):437-445

4 Artang R, Anderson M, Nielsen JD. Fully automated thromboelastograph TEG 6 s to measure anticoagulant effects of direct oral anticoagulants in healthy male volunteers. Res Pract Thromb Haemost 2019;3(03):391-396

5 Dias JD, Lopez-Espina CG, Ippolito J, et al. Rapid point-ofcare detection and classification of direct-acting oral anticoagulants with the TEG 6s: implications for trauma and acute care surgery. J Trauma Acute Care Surg 2019;87(02): 364-370

6 Godier A, Dincq AS, Martin AC, et al. Predictors of pre-procedural concentrations of direct oral anticoagulants: a prospective multicentre study. Eur Heart J 2017;38(31):2431-2439

7 Levy JH, Ageno W, Chan NC, Crowther M, Verhamme P, Weitz JISubcommittee on Control of Anticoagulation. When and how to use antidotes for the reversal of direct oral anticoagulants: guidance from the SSC of the ISTH. J Thromb Haemost 2016;14 (03):623-627

8 Seiffge DJ, Kägi G, Michel P, et al; Novel Oral Anticoagulants in Stroke Patients study group. Rivaroxaban plasma levels in acute ischemic stroke and intracerebral hemorrhage. Ann Neurol 2018; 83(03):451-459

9 Gosselin RC, Adcock DM, Bates SM, et al. International Council for Standardization in Haematology (ICSH) recommendations for laboratory measurement of direct oral anticoagulants. Thromb Haemost 2018;118(03):437-450

10 Deeks JJ, Altman DG. Diagnostic tests 4: likelihood ratios. BMJ 2004;329(7458):168-169

11 January CT, Wann LS, Calkins H, et al. 2019 AHA/ACC/HRS focused update of the 2014 AHA/ACC/HRS Guideline for the Management of Patients With Atrial Fibrillation: A Report of the American College of Cardiology/American Heart Association Task Force on Clinical Practice Guidelines and the Heart Rhythm Society in Collaboration With the Society of Thoracic Surgeons. Circulation 2019;140(02):e125-e151 
12 Hindricks G, Potpara T, Dagres N, et al. 2020 ESC Guidelines for the diagnosis and management of atrial fibrillation developed in collaboration with the European Association of Cardio-Thoracic Surgery (EACTS). Eur Heart J 2020;29:ehaa612

13 Testa S, Paoletti O, Legnani C, et al. Low drug levels and thrombotic complications in high-risk atrial fibrillation patients treated with direct oral anticoagulants. J Thromb Haemost 2018;16(05): 842-848
14 Eikelboom JW, Quinlan DJ, Hirsh J, Connolly SJ, Weitz JI. Laboratory monitoring of non-vitamin $\mathrm{K}$ antagonist oral anticoagulant use in patients with atrial fibrillation: a review. JAMA Cardiol 2017;2(05):566-574

15 Artang R, Anderson M, Riley P, Nielsen JD. Assessment of the effect of direct oral anticoagulants dabigatran, rivaroxaban, and apixaban in healthy male volunteers using a thrombin generation assay. Res Pract Thromb Haemost 2017;1(02):194-201 